\title{
Síndrome de distrés respiratorio agudo en paciente con COVID-19
}

Acute respiratory distress syndrome in patient with COVID-19

Síndrome do desconforto respiratório agudo em um paciente com COVID-19

Jesús Cleofas Ramírez-Campaña, ${ }^{*}$ Jesús Ariel Morales-Barraza, ${ }^{*}$ Cibeles Arrambí-Díaz, ${ }^{\ddagger}$

Lucia Concepción Valenzuela-Molina*

\section{RESUMEN}

Introducción: La enfermedad por coronavirus (COVID-19 coronavirus disease por sus siglas en inglés) es una emergencia sanitaria, y una de las complicaciones más temidas es el síndrome de distrés respiratorio agudo (SDRA) dada su elevada mortalidad.

Caso clínico: Paciente masculino de 59 años con antecedente de hipertensión y tabaquismo, que inicia síntomas posteriores a contacto con portador asintomático de COVID-19 proveniente del extranjero. La sintomatología que presentó fue mialgias, artralgias, febrícula de 37.7 grados, posteriormente fiebre de 38.4 grados, disnea, fatiga y odinofagia. Acude a consulta y se hospitaliza, otorgando tratamiento con cloroquina, azitromicina y oseltamivir por cuatro días; se tomó de muestra para COVID-19. El paciente mostró aumento de trabajo respiratorio, se tomó radiografía de tórax con opacidades heterogéneas periféricas de ambos pulmones y se corroboró por tomografía de tórax imagen de vidrio despulido. Presentó disnea progresiva e hipoxemia requiriendo manejo avanzado de la vía aérea y se trasladó a la Unidad de Cuidados Intensivos Metabólicos donde se recibió con ventilación mecánica (VM), requiriendo sedación, analgesia, relajante muscular así como ventilación protectora. Se realizaron cambios de posición para evitar microatelectasias. Se obtuvo por cultivos Pseudomonas aeruginosa y Escherichia coli. El día 11 de estancia en la UCI se logró progresar ventilación mecánica hasta destete de ésta, y el paciente se egresó de dicho servicio 48 horas después.

Conclusiones: El presente caso evidencia el progreso del daño pulmonar por COVID-19 causando falla respiratoria que requirió ventilación mecánica, el tratamiento crítico consistió en fortalecer la dinámica de calidad enfatizando monitoreo ventilatorio, hemodinámico y metabólico.

Palabras clave: COVID-19, síndrome de distrés respiratorio agudo (SDRA), ventilación mecánica (VM)

\section{ABSTRACT}

Introduction: Coronavirus disease (COVID-19) is a health emergency and one of the most feared complications is acute respiratory distress syndrome (ARDS) due to its high mortality.

Clinical case: A 59-year-old male patient with a history of hypertension and smoking, who begins to show symptoms after contact with an asymptomatic carrier of COVID-19 from abroad. The symptoms presented were myalgia, arthralgia, 37.7-degree fever, later 38.4-degree fever, dyspnea, fatigue and odynophagia. He went to the clinic and was hospitalized, being treated with chloroquine, azithromycin and oseltamivir for four days and a sample was taken for COVID-19. The patient presented increased respiratory work, chest radiography was taken with heterogeneous peripheral opacities of both lungs and was corroborated by chest tomography image of polished glass. He presented progressive dyspnea and hypoxemia requiring advanced airway management and was transferred to the metabolic intensive care unit where he was received with mechanical ventilation (MV), requiring sedation, analgesia, muscle relaxant, as well as protective ventilation. Changes of position were made to avoid micro atelectasis. It was obtained by culture of Pseudomonas aeruginosa and Escherichia coli. On the 11th day of the stay in ICU, mechanical ventilation was achieved until weaning, and the patient was discharged from ICU 48 hours later.

Conclusions: The present case evidences the progress of lung damage by COVID-19 causing respiratory failure requiring mechanical ventilation, where the critical treatment consisted in strengthening the quality dynamics emphasizing ventilatory, hemodynamic and metabolic monitoring.

* Hospital de Especialidades No. 2 del Instituto Mexicano del Seguro Social (IMSS). Cd. Obregón, Sonora.

‡ Hospital Regional No. 1 del IMSS. Cd. Obregón, Sonora.

Recepción: 18/05/2020. Aceptación: 27/05/2020.

www.medigraphic.com/medicinacritica
Keywords: COVID-19, acute respiratory distress syndrome (ARDS), mechanical ventilation (MV).

\section{RESUMO}

Introdução: A doença por coronavírus (COVID-19 coronavirus disease, por sus siglas en inglés) é uma emergência de saúde, e uma das complicações mais temidas é a síndrome do desconforto respiratório agudo (SDRA), dada sua alta mortalidade.

Caso clínico: Paciente do sexo masculino, 59 anos, com história de hipertensão e tabagismo, que iniciou os sintomas após contato com portador de COVID-19 assintomático do exterior. Os sintomas que apresentou foram mialgias, artralgias, febrícula de 37.7 graus, posterioriormente febre de 38.4 graus, dispnéia, cansaço e odinofagia, assiste a consulta médica e é hospitalizado, iniciando tratamento com cloroquina, azitromicina e oseltamivir durante 4 dias e foi uma colhida amostra por COVID-19. O paciente apresentava aumento do esforço respiratório, radiografia de tórax com opacidades periféricas heterogêneas de ambos pulmões e imagem em vidro fosco corroborada pela tomografia de tórax. Apresentou dispnéia progressiva e hipoxemia com necessidade de manejo avançado das vias aéreas e foi encaminhado para unidade de terapia intensiva metabólica onde recebeu ventilação mecânica (VM), necessitando de sedação, analgesia, relaxante muscular, além de ventilação protetora. Mudanças de posição foram feitas para evitar micro atelectasia. No cultivo obtivemos Pseudomonas aeruginosa e Escherichia coli. No 11 dia de internação na UTI, a ventilação mecânica foi progredida até o desmame, sendo dispensado do referido serviço 48 horas depois. Conclusões: O presente caso mostra a evolução do dano pulmonar por COVID-19 causando insuficiência respiratória que requer ventilação mecânica, onde o tratamento crítico consistiu no fortalecimento da dinâmica de qualidade com ênfase na monitoração ventilatória, hemodinâmica e metabólica.

Palavras-chave: COVID-19, síndrome do desconforto respiratório agudo (SDRA), ventilação mecânica (VM).

\section{INTRODUCCIÓN}

La enfermedad por coronavirus se describe por primera vez el 29 de diciembre de 2019 en la ciudad de Wuhan, provincia de Hubei, China, donde se identifica inicialmente como una «neumonía viral» y en enero de 2020 se identifica al patógeno en el documento «Novel coronavirus 2019», a fin del mismo mes la OMS (Organización Mundial de la Salud) declaró emergencia sanitaria; sin embargo, es en febrero cuando se nombra oficialmente «enfermedad de coronavirus 2019».1

La neumonía COVID-19 es una enfermedad específica con fenotipos peculiares, aunque puede cumplir con la definición de la SDRA de Berlín (Tabla 1), ${ }^{2}$ su principal característica es la disociación entre la gravedad de la hipoxemia y el mantenimiento de una mecánica respiratoria relativamente buena. ${ }^{3}$

El periodo de incubación se definió como el intervalo entre la fecha potencial de contacto más temprana de la fuente de transmisión y la fecha más temprana de aparición de los síntomas, y éstos pueden aparecer desde el segundo hasta el día 12 posterior a la exposición. ${ }^{4}$ 
Tabla 1: Criterios de Berlín para síndrome de distrés respiratorio agudo.

Infiltrados pulmonares bilaterales en estudio de imagen (radiografía de tórax, tomografía computarizada o ultrasonido pulmonar)

Edema pulmonar no justificado principalmente por falla cardiaca (evidenciado de forma objetiva, ej. ecocardiografía)

$\mathrm{PaO}_{2} / \mathrm{FiO}_{2}<300$ (con PEEP/CPAP $\geq 5 \mathrm{cmH}_{2} \mathrm{O}$ )

Afección pulmonar de inicio menor de siete días

$\mathrm{PaO}_{2} / \mathrm{FiO}_{2}=$ presión alveolar de oxígeno/fracción inspirada de oxígeno; $\mathrm{PEEP}=$ presión positiva al final de la espiración; CPAP = presión positiva continua en las vías respiratorias; $\mathrm{CmH}_{2} \mathrm{O}=$ centímetro de agua.

La ruta de transmisión de este virus es por contacto, gotitas de Flügge y aerosoles. Las manifestaciones clínicas descritas son fiebre, tos, odinofagia, cefalea, fiebre, mialgias, artralgias, en casos leves, mientras que en casos graves se presenta disnea progresiva, incluso síndrome de dificultad respiratoria aguda, así como afección a otros órganos con insuficiencia renal aguda e insuficiencia cardiaca. ${ }^{4}$

La base del diagnóstico del COVID-19 es la prueba de reacción en cadena de polimerasa en tiempo real (RT-PCR por sus siglas en inglés, reverse transcriptase-polymerase chain reaction) que incluyen hisopado orofaríngeo, nasofaríngeo, esputo, lavado bronco alveolar y aspirados traqueales. ${ }^{4,5}$

Hasta la fecha no hay un tratamiento específico y probado contra la infección, se recomienda de forma inmediata establecer las medidas de prevención y control, con una monitorización estrecha; en los casos leves se puede dar manejo ambulatorio y en los casos graves proporcionar tratamiento de soporte respiratorio y orgánico. 6,7

\section{CASO CLÍNICO}

Se trata de paciente masculino de 59 años, residente y originario de Sonora, casado, católico, estudios de postgrado y trabajador de la salud, dentro de los antecedentes crónico degenerativos refiere hipertensión arterial de 12 años de evolución en tratamiento con losartán; y tabaquismo, el cual fue suspendido 12 años atrás, niega alergias y hospitalizaciones previas.

Inicia padecimiento posterior a tener contacto previo con personas provenientes de España, con inicio de sintomatología el día 18 de marzo de 2020 cuando presenta mialgias, artralgias, febrícula de 37.7 grados, posteriormente fiebre de 38.4 grados, disnea, fatiga y odinofagia; acude a consulta y se hospitaliza, otorgando tratamiento con cloroquina, azitromicina y oseltamivir por cuatro días y se tomó de muestra para COVID-19.

El paciente presentó aumento de trabajo respiratorio, se tomó radiografía de tórax (Figura 1) con opacidades heterogéneas periféricas de ambos pulmones, comportándose como neumonía por COVID-19 y se solicitó tomografía simple de tórax (TC) donde se observa imagen de vidrio despulido en las periferias pulmonares, zonas de consolidación y atelectasias (Figura 2). Los laboratorios iniciales con leucocitos de 11,200, neutrófilos de $88 \%$ y linfocitos de $8 \%$, hemoglobina $(\mathrm{Hb}) 14 \mathrm{~g} / \mathrm{dL}$, hematocrito (Hto) $42.2 \%$, plaquetas de 359,000 , PCR 96, DHL de 684. Se recaba prueba RT-PCR positiva, se agrega disnea progresiva e hipoxemia requiriendo manejo avanzado de la vía aérea.

Se traslada a la Unidad de Cuidados Intensivos Metabólicos donde se recibe con ventilación mecánica (VM) con los siguientes parámetros ventilatorios, variable control: presión, secuencia respiratoria: mandataria continua, estrategia: fija, $\mathrm{FiO}_{2} 80 \%$, presión inspiratoria: 10 , Ti 1.3 segundos, FR 16, PEEP 10, Rampa 0.3, con índice de Kirby de $83 \mathrm{mmHg}$, su gasometría con $\mathrm{pH} 7.10, \mathrm{pCO}_{2} 82$, $\mathrm{PO}_{2} 47, \mathrm{SO}_{2} 65 \%, \mathrm{HCO}_{3}$ 25.5. Los laboratorios al ingresar con leucocitos en 22,000 , linfocitos $18.3 \%$, neutrófilos $72 \%$, Hb $13.2 \mathrm{~g} / \mathrm{dL}$, Hto $40 \%$, plaquetas de 698,000, fibrinógeno 1,132 , troponina I ultrasensible 8.6 , creatinfosfoquinasa (CPK) 40.10, colesterol inicial 185, control de 305, dímero D 50 inicial, control de 830 (Tabla 2).

Durante su estancia en la $\mathrm{UCl}$ se mantiene con sedación y analgesia, en los primeros cuatro días se utilizó relajante muscular por hipoxemia refractaria en la VM se utilizó ventilación protectora con volumen tidal a 6 $\mathrm{ml} / \mathrm{kg}$ de peso predicho, cuidando presión plateau $<30$ $\mathrm{mmH}_{2} \mathrm{O}$ ideal $<28 \mathrm{mmH}_{2} \mathrm{O}$ (Tabla 3). Para reducir el daño pulmonar por VM se aplicaron cambios de posición constantemente y así evitar microatelectasias, al mismo tiempo que el uso de heparina de bajo peso molecular a dosis de anticoagulación. Se realizaron cultivos de orofaringe para no abrir circuito y causar aumen-

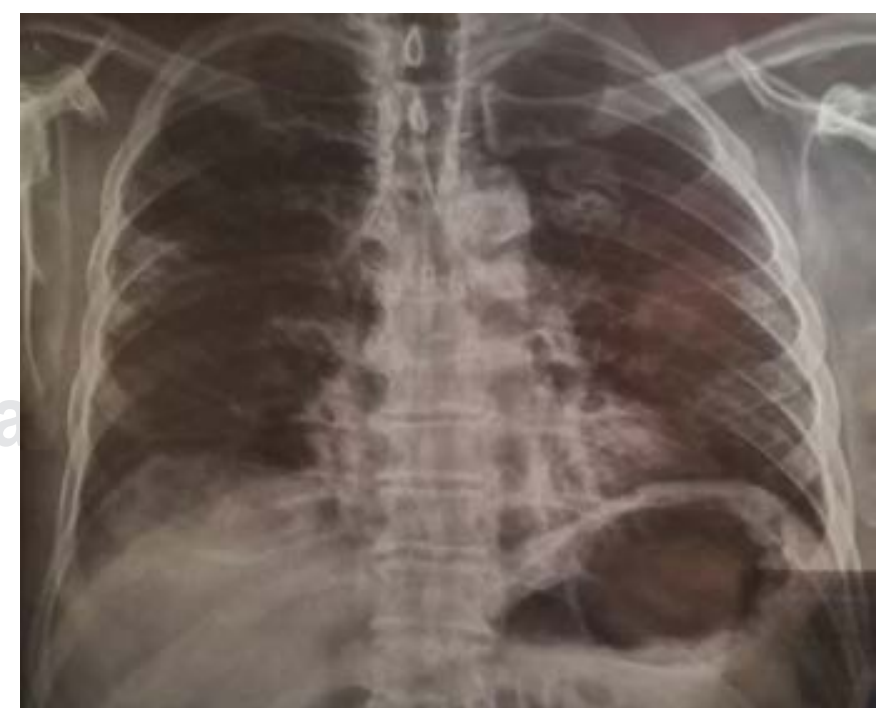

Figura 1: Radiografía simple de tórax donde se observan opacidades heterogéneas periféricas de ambos pulmones. Día del estudio 27 de marzo de 2020. 

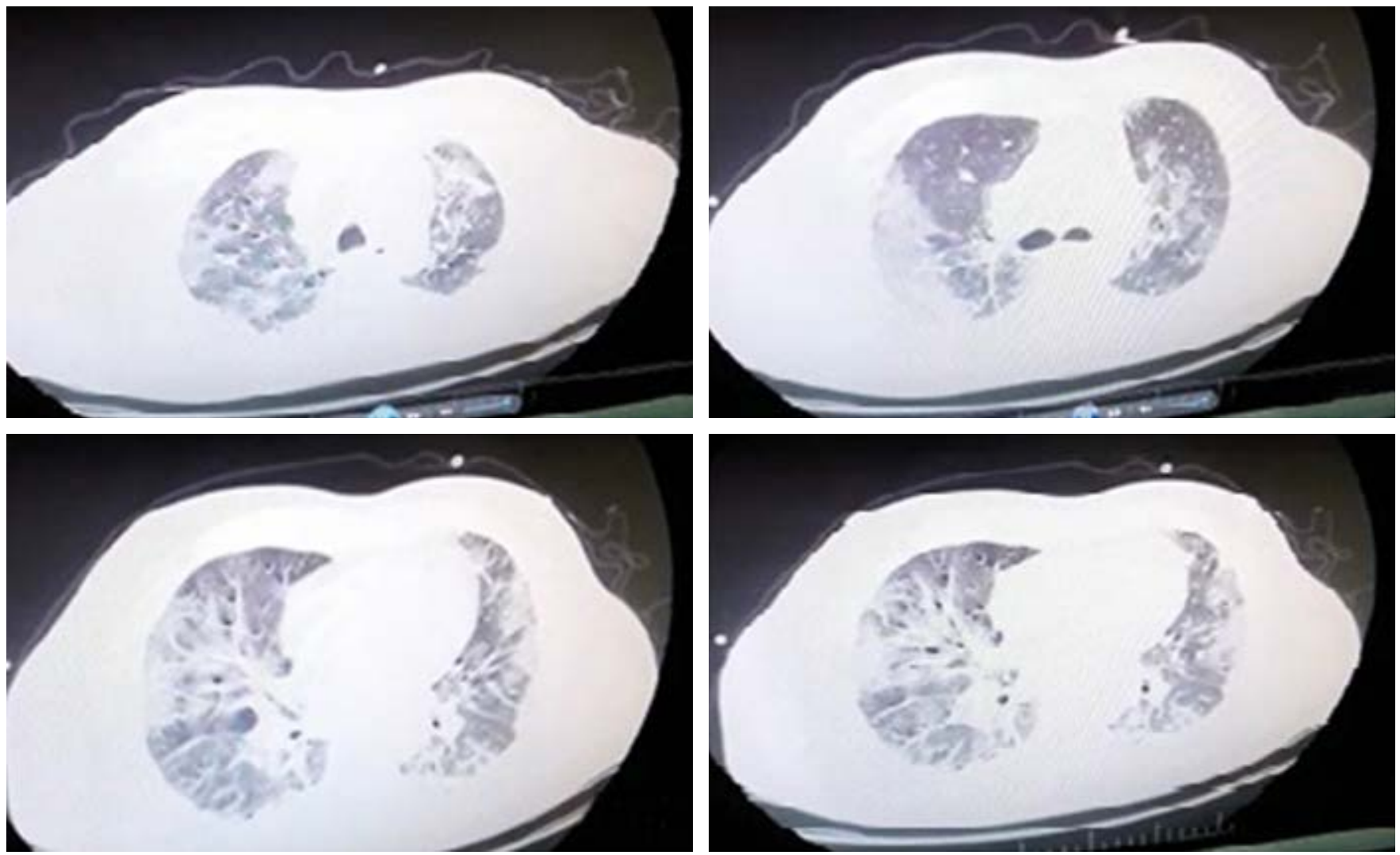

Figura 2: Tomografía de tórax que muestra imagen de vidrio despulido de distribución periférica, zonas de consolidación así como atelectasias. Día del estudio 28 de marzo de 2020.

to de aerosoles, y se obtuvo desarrollo $>100,000$ UEC de Pseudomonas aeruginosa, y pasado el periodo de mayor contagio (14 días) se tomó cultivo de secreción bronquial que reportó Escherichia coli multidrogorresistente BLEE positivo sensible a tigeciclina.

Referente al soporte circulatorio el paciente cursó con choque séptico, utilizando dosis tituladas de noradrenalina para mantener perfusión y garantizar la microcirculación, así como glucocorticoides de tipo hidrocortisona hasta completar el ciclo recomendado a dosis de $200 \mathrm{mg}$ cada 24 horas.

Posterior al retiro del relajante muscular, y al disminuir dosis de sedantes se inició rehabilitación temprana del paciente. En el día 11 de estancia en la UCI se logró progresar ventilación mecánica hasta destete de ésta, egresándose de dicho servicio dos días después.

El presente caso evolucionó de leve-moderado-severo-crítico, donde progresa el daño pulmonar causando falla respiratoria que requirió ventilación mecánica, y el tratamiento crítico consistió en fortalecer la dinámica de calidad en nuestra unidad, asegurar un aporte calórico adecuado (25 Kcal $/ \mathrm{kg}$ ), mantener un balance de líquidos y electrolitos adecuado para mantener la estabilidad metabólica, monitorear signos vitales, saturación de oxígeno y paraclínicos.
Tabla 2: Laboratorios desde el inicio de la enfermedad.

\begin{tabular}{|c|c|c|c|}
\hline Reactivos (UM) & Inicio & $\begin{array}{c}\text { Día } 1 \text { en } \\
\text { UCl }\end{array}$ & $\begin{array}{c}\text { Día } 10 \text { en } \\
\text { UCl }\end{array}$ \\
\hline $\mathrm{Hb}(\mathrm{mg} / \mathrm{dL})$ & 14.1 & 13.21 & 12.39 \\
\hline Hematocrito (\%) & 42 & 40.3 & 37.9 \\
\hline Leucocitos $\left(10^{3} / \mu \mathrm{L}\right)$ & 11,200 & 22.8 & 10.4 \\
\hline Linfocitos (\%) & 8 & 18.3 & 54.3 \\
\hline Neutrófilos (\%) & -- & 72.5 & 54.3 \\
\hline Plaquetas $\left(\times 10^{3} / \mu \mathrm{L}\right)$ & 352 & 698.1 & 515.1 \\
\hline Fibrinógeno (mg/dL) & -- & $1,132.4$ & 981.27 \\
\hline TP (seg) & -- & 16.9 & 15.3 \\
\hline TPT (seg) & -- & 32.3 & 33.6 \\
\hline Dímero D (ng/mL) & 0.3 & 50 & 830 \\
\hline FA (UI/L) & 136 & 79 & 326 \\
\hline DHL (UI/L) & 684 & 451 & 513 \\
\hline TGO (UI/L) & 72 & 78 & 55 \\
\hline TGP (UI/L) & 70 & 80 & 66 \\
\hline CPK (UI/L) & -- & 147 & 131 \\
\hline CPK-mb (U/L) & -- & 40 & 25 \\
\hline Troponina I HS & -- & 8.6 & -- \\
\hline PCR (mg/L) & 96 & 17.1 & 10.8 \\
\hline Glucosa(mg/dL) & 107 & 164 & 78 \\
\hline Colesterol & & 185 & 305 \\
\hline
\end{tabular}

UM = unidades de medida; $\mathrm{UCI}=$ Unidad de Cuidados Intensivos; $\mathrm{Hb}=$ hemoglobina; $\mathrm{TP}=$ tiempo de protrombina; TPT = tiempo de tromboplastina parcial; $\mathrm{FA}=$ fosfatasa alcalina; $\mathrm{DHL}=$ deshidrogenasa láctica; $\mathrm{TGO}$ = transaminasa glutámico oxalacética; TGP = transaminasa glutámico-pirúvica; $\mathrm{CPK}$ : creatinfosfoquinasa; $\mathrm{CPK}$-mb: creatinfosfoquinasa fracción $\mathrm{mb} ; \mathrm{PCR}=$ proteína $\mathrm{C}$ reactiva. $\mathrm{HS}=$ ultrasensible Datos obtenidos del expediente. 


\section{DISCUSIÓN}

A inicios de diciembre de 2019 en Wuhan, capital de la provincia de Hubei, se presentan los primeros casos de neumonía de origen desconocido. El patógeno se identificó y nombró coronavirus del síndrome respiratorio agudo 2 (SARS-CoV-2), ya que se encontró una similitud filogenética con el SARS-CoV. Dada su rápida propagación, la Organización Mundial de la Salud (OMS) llamó recientemente a la enfermedad coronavirus 2019 (COVID-19). 1,4,6

EI SARS-CoV-2 es un coronavirus $\beta$, que está envuelto en un virus ARN de sentido positivo no segmentado que pertenece a la subfamilia Orthocoronavirinae, al subgénero Sarbecovirus; se caracteriza por ser un virus ARN de cadena positiva que bajo microscopia electrónica posee una apariencia de corona. Recientemente se descubrió que este nuevo coronavirus tiene gran afinidad por el receptor de enzima convertidora de angiotensina-2 (ACE-2) con la que establece una unión covalente máxima y que tiene una gran expresión en el neumocito. $^{7}$
De acuerdo con la recomendación más reciente, la enfermedad por coronavirus 2019 se clasifica en cuatro tipos: casos leves, ordinarios, graves y críticos. Los casos leves y ordinarios tienen buen pronóstico, pero los casos graves, especialmente los críticos, siempre presentan una disfunción orgánica sistemática y una hospitalización prolongada con una recuperación deficiente. ${ }^{8}$

Las evaluaciones de laboratorio consistieron en un conteo sanguíneo completo, análisis químico sanguíneo, pruebas de coagulación, evaluación de la función hepática y renal, y medidas de electrolitos, procalcitonina y creatina quinasa; considerando a la proteína C reactiva, lactato deshidrogenasa y los glóbulos blancos como predictor de gravedad. ${ }^{5,9}$

En cuanto a los estudios de gabinete, las imágenes hasta hoy características de neumonía viral por COVID-19 son infiltrados heterogéneos bilaterales, periféricos en la radiografía de tórax, mientras que en la TC se observan opacidades con atenuación de vidrio esmerilado periférico, focal o multifocal, y bilaterales en $50-75 \%$ de los casos. Al progresar la enfermedad entre los días 9 y 13, aparecen lesiones en patrón de mosaico

Tabla 3: Monitoreo ventilatorio en Unidad de Cuidados Intensivos.

\begin{tabular}{|c|c|c|c|c|c|c|c|c|c|c|c|c|c|c|c|}
\hline Día/hora & $1 / 10: 20$ & 2/10:25 & $3 / 10: 30$ & 4/13:00 & 5/09:00 & 6/08:50 & $7 / 7: 00$ & 8/09:00 & 9/09:50 & $10 / 10: 25$ & $11 / 10: 30$ & 12 & 13 & 14 & 15 \\
\hline $\begin{array}{l}\text { Modo } \\
\text { ventilación }\end{array}$ & $\mathrm{A} / \mathrm{CV}$ & $\mathrm{A} / \mathrm{CV}$ & $\mathrm{A} / \mathrm{CV}$ & $\mathrm{A} / \mathrm{CV}$ & $\mathrm{A} / \mathrm{CP}$ & $\mathrm{A} / \mathrm{CP}$ & $\mathrm{A} / \mathrm{CP}$ & CPAP & CPAP & CPAP & CPAP & $\begin{array}{l}\text { Ret. } \\
\text { tubo }\end{array}$ & Esp. & Esp. & Alta \\
\hline PEEP & 8 & 8 & 8 & 8 & 8 & 8 & 8 & 8 & 8 & 8 & 8 & --- & --- & --- & --- \\
\hline $\mathrm{FiO}_{2} \%$ & $65 \%$ & $40 \%$ & $40 \%$ & $45 \%$ & $40 \%$ & 40 & $35 \%$ & $30 \%$ & $27 \%$ & 27 & 21 & 21 & 30 & 25 & 21 \\
\hline SAT VC & $75 \%$ & & $80 \%$ & & $72 \%$ & $66 \%$ & & $80 \%$ & $83 \%$ & 68 & & --.- & -- & -.- & --- \\
\hline Relajado & Sí & Sí & Sí & Sí & Sí & No & No & No & No & No & No & ---.- & --- & --- & --- \\
\hline VT & 430 & 420 & 450 & 450 & 500 & 530 & $422 / 379$ & 530 & 580 & 606 & 626 & ---- & --- & --- & --- \\
\hline VM & 6.8 & 6.3 & 6.8 & 7.19 & 8.5 & 8.8 & 7.18 & 8.1 & 8.0 & 9.9 & 11.4 & ---- & --- & --- & --- \\
\hline $\mathrm{R}$ & 8 & 8.5 & 8.2 & & & 9.1 & & 9.4 & 9 & 10.6 & 7.9 & ---- & --- & ---- & --- \\
\hline P. pico & 23 & 25 & 21 & 24 & 22 & 24 & 23 & 22 & 21 & 24 & 19 & ---- & ---- & ---- & ---- \\
\hline P. meseta & 16 & 23 & 22 & 22 & 22 & 22 & 18 & 17 & 16 & 19 & 15 & ---- & --- & --- & --- \\
\hline P. media & 13 & 12 & 12 & 11 & 13 & 13 & 12 & 12 & 13 & 13 & 11 & ---- & --- & --- & --- \\
\hline $\mathrm{FR}$ & 16 & 15 & 17 & 16 & 16 & 18 & 18 & 18 & 17 & 16 & 18 & 20 & 23 & 25 & 22 \\
\hline I:E & $1: 2.4$ & 1.23 & $1: 2.2$ & $1: 2.4$ & $1: 1.9$ & $1: 2$ & $1: 2$ & $1: 2$ & $1: 2$ & $1: 2.6$ & $1: 2$ & --- & ---- & $---\cdot$ & --- \\
\hline $\mathrm{pH}$ & 7.27 & 7.31 & 7.36 & 7.44 & 7.52 & 7.51 & 7.45 & 7.44 & 7.40 & 7.45 & 7.40 & 7.40 & 7.39 & 7.41 & 7.40 \\
\hline $\mathrm{PaO}_{2}$ & 70 & 120 & 61 & 74 & 67 & 65 & 70 & 81 & 71 & 83 & 67 & ---- & --- & --- & ---- \\
\hline $\mathrm{pCO}_{2} / \mathrm{HCO}_{3}$ & $68 / 28$ & $67 / 30.3$ & $64 / 36$ & $55 / 40$ & $50 / 40$ & $50 / 36.6$ & $52 / 36.1$ & $48 / 30$ & $45 / 27$ & $36 / 25$ & $44 / 27.36$ & 24 & --- & ---- & 24 \\
\hline Saturación \% & 93 & 98 & 94 & 95 & 95 & 94 & 95 & 95 & 96 & 97 & 93 & 96 & 95 & 96 & 95 \\
\hline IK: & 116 & 168 & 152 & 164 & 167 & 162 & 200 & 212 & 256 & 307 & 316 & 300 & --- & ---- & --- \\
\hline Gaa: & 313 & 80.6 & 142 & 171.25 & 154 & 156 & 113 & 85 & 56 & 63 & 26 & -----. & --- & ---. & --- \\
\hline QSQT: & 17 & 4.4 & 7.8 & 9.5 & 8.5 & 8.6 & 6.2 & 5 & 3.1 & 13.4 & 1.4 & ---- & --- & --- & --- \\
\hline DD: & 24 & 28 & 55 & 22.5 & 34.7 & 30 & 28 & 35 & 41 & 32.6 & 56 & ----- & --- & ---. & --- \\
\hline Peso & 91.5 & 97.5 & 95 & 98 & 98.5 & 105 & 96.5 & 96 & 95 & 96 & 91.5 & 95 & 94 & 92 & 88 \\
\hline $\begin{array}{l}\text { Driving } \\
\text { pressure/R }\end{array}$ & 15 & 15 & 14 & 12 & 14 & 14 & 10 & 9.5 & 9 & 11 & 7 & --- & --- & --- & --- \\
\hline $\begin{array}{l}\text { Poder } \\
\text { mecánico }\end{array}$ & 2.9 & 6.1 & 7 & 11.2 & 10 & 9.3 & 11.5 & 8 & 8 & 11.5 & 11.7 & ---- & --- & ---- & --- \\
\hline $10 x$ & 12 & 4 & 6 & 9 & 7 & 8 & 7 & 7 & 6 & 3.5 & 3.9 & ---- & --- & --- & --- \\
\hline TAM & 83 & 78 & 90 & 90 & 90 & 80 & 80 & 85 & 81 & 78 & 90 & 85 & 80 & 82 & 85 \\
\hline PVC & 14 & 15 & 14 & - & 13 & 10 & 9 & 9 & 8 & 7 & 8 & 10 & 11 & 9 & 10 \\
\hline $\mathrm{FC}$ & 99 & 73 & 68 & 60 & 50 & 74 & 69 & 80 & 77 & 89 & 77 & 66 & 68 & 70 & 66 \\
\hline Temp. $^{\circ}$ & 37.0 & 35.9 & 36.2 & 36.4 & 36.2 & 36.8 & 36.8 & 36.5 & 36 & 36.8 & 37.0 & 36 & 36 & 36 & 36 \\
\hline
\end{tabular}

$\mathrm{A} / \mathrm{CV}=$ asisto control volumen; $\mathrm{A} / \mathrm{CP}$ = asisto control presión; $\mathrm{CPAP}=$ presión positiva continua en las vías respiratorias; Ret. tubo = retiro de tubo (cánula orotraqueal); Esp. = respiración espontánea; $\mathrm{PEEP}$ = presión positiva al final de la espiración; $\mathrm{FiO}_{2}$ = fracción inspirada de oxígeno; $\mathrm{SAT} \mathrm{VC}=$ saturación venosa central; $\mathrm{VT}=$ volumen tidal; $\mathrm{VM}=$ volumen minuto; $\mathrm{R}=$ resistencia de la vía aérea; $\mathrm{P}$. pico= presión pico; $\mathrm{P}$. meseta $=$ presión meseta; $\mathrm{P}$. media = presión media $\mathrm{FR}=$ frecuencia respiratoria; $\mathrm{I}: \mathrm{E}=$ inspiración:espiración; $\mathrm{pH}=$ medida de acidez 0 alcalinidad; $\mathrm{PaO}{ }_{2}=$ presión alveolar de oxígeno; $\mathrm{PCO}_{2} / \mathrm{HCO}_{3}$ = presión parcial de dióxido de carbono/Bicarbonato; IK = índice de Kirby o de oxigenación; Gaa = gradiente alvéolo arterial; $\mathrm{QSQT}=$ shunt pulmonar; $\mathrm{DD}=$ distensibilidad dinamica; Driving pressure $/ \mathrm{R}$ = presión de conducción; IOX = índice de oxigenación; TAM = tensión arterial media; $P V C=$ presión venosa central; FC = frecuencia cardiaca, Temp. = temperatura. 
de pavimentación, dichas lesiones desaparecen en un mes o más. Sin embargo, la ACR (American College of Radiology por sus siglas en inglés) sugiere que no se debe usar la tomografía computarizada para detectar o como prueba de primera línea para diagnosticar COVID-19, ya que las imágenes encontradas no son específicas para dicha patología, siendo la prueba viral el único método específico de diagnóstico. ${ }^{10,11}$

En cuanto al tratamiento, los pacientes con neumonía COVID-19, que cumplen los criterios de Berlín para el SDRA, presentan una forma atípica del síndrome, por ello el tratamiento ventilatorio en este caso se apegó a lo referido por Karbing y colaboradores, ${ }^{12}$ en el que se identifica que el uso de PEEP en pacientes con SDRA puede tener efectos tanto benéficos como perjudiciales, ya que una PEEP alta en un pulmón poco reclutable tiende a resultar en un severo deterioro hemodinámico, hipoxemia, aumento de cortocircuito y retención de líquidos, por lo tanto se debe individualizar la estrategia ventilatoria y tratar de descubrir qué efecto tendrá el PEEP en el pulmón a ventilar, en este caso encontramos que el uso de PEEP elevado ( $>10$ $\mathrm{cmH}_{2} \mathrm{O}$ ) perjudica la ventilación y deteriora su distensibilidad encontrando que el valor ideal para este pulmón es de $8 \mathrm{~cm} \mathrm{H}_{2} \mathrm{O}$, en el que se obtiene mejor ventilación y disminución de corto circuitos pulmonares, ya que el movimiento del volumen es diferente en las distintas regiones pulmonares según sea el daño en dicho sitio pulmonar ya establecido, con gran beneficio en metas de protección pulmonar, y así lograr un mejor equilibrio entre el intercambio de $\mathrm{O}_{2}$ y preservar el intercambio de $\mathrm{CO}_{2}$ (ventilación) en la distensibilidad, no impactando de manera negativa en la frecuencia cardiaca, tensión arterial, saturación de oxígeno y presión plateau. ${ }^{11}$

\section{CONCLUSIÓN}

EI SDRA en el paciente con COVID-19 es una entidad nueva, aún de prevalencia inespecífica. En México actualmente nos encontramos en la fase de contingencia cuya mortalidad hoy es de $10.7 \%$. En este trabajo se pretende registrar el manejo que se otorgó en SDRA por SARS-CoV-2 y mostrar que la presentación clínica fue similar a lo reportado en la bibliografía; sin embargo, en el tratamiento se priorizó en metas de protección pulmonar y se obtuvo una evolución exitosa. El pronóstico en la mayoría de los casos es incierto, aunque si el abordaje es precoz y oportuno, la evolución puede ser favorable.

\section{AGRADECIMIENTOS}

Se extiende un agradecimiento al Instituto Mexicano del Seguro Social y particularmente al personal de salud de la Unidad de Cuidados Intensivos Metabólicos del Hospital de Especialidades No. 2.

\section{BIBLIOGRAFÍA}

1. Jin YH, Cai L, Cheng ZS, Cheng H, Deng T, Fan YP, et al. A rapid advice guideline for the diagnosis and treatment of 2019 novel coronavirus (2019-nCoV) infected pneumonia (standard version). Mil Med Res [Internet]. 2020;7(1):4. Available in: https:// doi.org/10.1186/s40779-020-0233-6

2. Bernard GR, Artigas A, Brigham KL, et al. The AmericanEuropean Consensus Conference on ARDS. Definitions, mechanisms, relevant outcomes, and clinical trial coordination. Am J Respir Crit Care Med. 1994;149(3 Pt 1):818-824.

3. Gattinoni L, Chiumello D, Rossi S. COVID-19 pneumonia: ARDS or not? Crit Care. 2020;24(1):154. doi: 10.1186/s13054-02002880-z

4. Guan WJ, Ni ZY, Hu Y, et al. Clinical characteristics of coronavirus disease 2019 in China. N Engl J Med. 2020;382(18):1708-1720. doi: 10.1056/NEJMoa2002032.

5. Sharfstein JM, Becker SJ, Mello MM. Diagnostic testing for the novel coronavirus [published online ahead of print, 2020 Mar 9]. JAMA. 2020; 10.1001/jama.2020.3864. doi: 10.1001/ jama.2020.3864.

6. Aragón-Nogales R, Vargas-Almanza I, Miranda-Novales MG. COVID-19 por SARS-CoV-2: la nueva emergencia de salud. Rev Mex Pediatr. 2019;86(6):213-218. https://doi.org/10.35366/91871

7. Martínez-Portilla RJ, Torres-Torres J, Gurrola R, Moreno-Uribe N. Guía mexicanas de SARS-CoV-2 y embarazo. 2020. doi: 10.17605/OSF.IO/GDV98.

8. Wang Y, Liu Y, Liu L, Wang X, Luo N, Li L. Clinical outcomes in 55 patients with severe acute respiratory syndrome coronavirus 2 who were asymptomatic at hospital admission in Shenzhen, China. J Infect Dis. 2020;221(11):1770-1774. doi: 10.1093/infdis/ jiaa119.

9. Gattinoni L, Coppola S, Cressoni M, Busana M, Rossi S, Chiumello D. COVID-19 does not lead to a "typical" acute respiratory distress syndrome. Am J Respir Crit Care Med. 2020;201(10):1299-1300. doi: 10.1164/rccm.202003-0817LE.

10. American College of Radiology. Recommendations for the use of Chest Radiography and Computed Tomography (CT) for Suspected COVID-19 Infection. 2020. Available in: https://www. acr.org/Advocacy-and-Economics/ACR-Position-Statements/ Recommendations-for-Chest- Radiography-and-CT-forSuspected-COVID19-Infection

11. Ye Z, Zhang Y, Wang Y, Huang Z, Song B. Chest CT manifestations of new coronavirus disease 2019 (COVID-19): a pictorial review. Eur Radiol. 2020;30(8):4381-4389.

12. Karbing DS, Panigada M, Bottino N, et al. Changes in shunt, ventilation/perfusion mismatch, and lung aeration with PEEP in patients with ARDS: a prospective single-arm interventional study. Crit Care. 2020;24(1):111. doi: 10.1186/s13054-020-2834-6.

Conflicto de intereses: Los autores declaran no tener conflicto de intereses.

\author{
Correspondencia: \\ Dra. Cibeles Arrambí-Díaz \\ Departamento de Urgencias del \\ Hospital Regional No. 1 del IMSS. \\ Guerrero y Prolongación Hidalgo S/N, \\ Col. Bella Vista, 85130, \\ Cajeme, Ciudad Obregón, Sonora. \\ Tel: (644) 1392667 \\ E-mail: cibelesarrambi@hotmail.com
}

